\title{
Des pleurs ou des coups
}

Affects et relations dans l'initiation au bwete misoko (Gabon)

Tears and shocks. Affects and relations during initiation to the bwete misoko (Gabon)

\section{Julien Bonhomme}

\section{OpenEdition \\ Journals}

Édition électronique

URL : http://journals.openedition.org/span/785

DOI : $10.4000 /$ span.785

ISSN : 2268-1558

Éditeur

École pratique des hautes études. Sciences humaines

\section{Édition imprimée}

Date de publication : 1 décembre 2008

Pagination : 133-163

ISSN : 0294-7080

Référence électronique

Julien Bonhomme, "Des pleurs ou des coups », Systèmes de pensée en Afrique noire [En ligne],

18 | 2008, mis en ligne le 05 juin 2013, consulté le 02 mai 2019. URL : http://journals.openedition.org/ span/785 ; DOI : 10.4000/span.785

(c) École pratique des hautes études 



\title{
Des pleurs ou des coups \\ Affects et relations dans l'initiation au bwete misoko (Gabon)
}

\author{
Julien Bonhomme* \\ Maître de conférences \\ Université Lyon-2 \\ détaché comme directeur-adjoint du dépt. \\ de la recherche et de l'enseignement \\ Musée du Quai Branly
}

\begin{abstract}
Par affect, j'entends les affections du corps, qui augmentent ou diminuent, aident ou contrarient, la puissance d'agir de ce corps, et en même temps les idées de ces affections.

Spinoza, Éthique, troisième partie, définition III.
\end{abstract}

Aux déclarations du devin concernant son affliction, le patient se met à pleurer. À l'issue de cette séance divinatoire, il accepte de se faire initier au bwete misoko en mangeant les racines hallucinogènes d'eboga afin de voir par lui-même l'origine de son infortune et d'y trouver un remède. Pendant cette veillée initiatique, aux premières visions angoissantes, il se met de nouveau à sangloter. Les initiateurs le réprimandent aussitôt, le pressant de cesser les pleurs et de se reprendre. Plus tard au cours de la nuit, il parvient dans ses visions à identifier le sorcier responsable de son infortune et à le frapper à mort, mimant réellement les coups avec son chasse-mouches sous les applaudissements de l'assistance. L'initiation aura finalement été réussie.

Dans le présent article, j'analyse le processus initiatique du bwete misoko - l'un des principaux rites initiatiques de guérison pratiqués au Gabon - en me focalisant sur sa dimension affective. Le rituel constitue en effet avant tout une expérience vécue, impliquant des acteurs, des interactions mais aussi des émotions. Ainsi, les initiations font souvent un usage productif de la douleur, de la terreur ou de la honte à travers les brimades ${ }^{1}$. De même, les rites d'affliction

\footnotetext{
* Je tiens à remercier François Berthomé et Michael Houseman pour leurs critiques constructives.

1 Houseman, 1986; Whitehouse, 2000.
} 
2 Lévi-Strauss, 1958a, 1958b; Favret-Saada, 1977, 1985; Kapferer, 1997.

${ }^{3}$ Pour une analyse critique de ce modèle cathartique, $c f$. Berthomé, 2006.

${ }^{4}$ Gluckman, 1963 sur les rites de rébellion; Lewis, 1971 sur les rites de possession.

${ }^{5}$ Turner, 1968, 1969.

${ }^{6}$ Scheff, 1977, 1979.

${ }^{7}$ Lévi-Strauss, 1958a, 1958b.

8 Je m'intéresse donc moins au soubassement affectif qui sous-tend toute l'existence qu'à l'expression spécifique des émotions en contexte rituel. opèrent habituellement un travail sur la souffrance, l'angoisse ou le désarroi dans la mesure où ceux-ci constituent une dimension essentielle du malheur ${ }^{2}$. Cette importance centrale des émotions illustre bien la performativité du rituel : il fait quelque chose plus encore qu'il ne dit quelque chose - l'expression des affects étant la manifestation tangible de cette opération. Comme l'incipit le montre en raccourci, dans le cas du bwete misoko, l'initiation opère en effet un travail sur les affects du novice: les pleurs du patient lors de la séance divinatoire ne sont plus acceptables lors de l'initiation, et doivent laisser place à une riposte agressive contre le responsable supposé de l'affliction.

L'analyse anthropologique des émotions en contexte rituel obéit le plus souvent à un modèle cathartique ${ }^{3}$. Le rituel constitue ainsi un exutoire pour soulager les tensions et les émotions menaçant l'ordre social ${ }^{4}$, un instrument de canalisation et de socialisation des émotions ${ }^{5}$, une catharsis opérant une mise à distance des émotions ${ }^{6}$, une abréaction permettant d'intégrer une expérience affective informe dans une forme culturelle stable? L'émotion est conçue comme une énergie réprimée, une tension qui ne trouve pas à s'extérioriser. À travers la manipulation de symboles évocateurs, le rituel permet alors à ces tensions de s'exprimer publiquement. Cette décharge émotionnelle libératrice confère ainsi au rituel une fonction homéostatique (que celle-ci soit pensée en termes d'ordre social ou d'ordre symbolique). Dans cet article, j'entends dépasser, ou du moins approfondir, ce modèle cathartique classique. Je souhaite en effet montrer que le rituel opère moins une décharge qu'une production et une transformation des affects. Les affects ne doivent pas être envisagés comme de simples tensions qui préexistent au rituel, mais comme des phénomènes qualitativement distincts qui émergent du processus rituel et sont transformés par lui ${ }^{8}$.

Cette transformation des affects constitue l'un des aspects sensibles de la transformation initiatique. L'initiation opère en effet une véritable transformation de la personne, ce qu'exprime si communément l'idéologie de la renaissance initiatique. J'ai montré ailleurs comment, 
dans le bwete misoko, cette opération passe par la transformation complexe des relations entre profane et initié, initiateur et initié, hommes et femmes, aîné et cadet, ancêtres et vivants, devin et patient, sorcier et victime, père et fils, mère et enfant ${ }^{9}$. À travers une série d'interactions rituelles spécifiques, le réseau relationnel dans lequel l'initié se trouve inséré est ainsi progressivement remanié, décomposé et recomposé ${ }^{10}$. Or, ce travail initiatique sur les relations est corrélativement un travail sur les affects, dans la mesure où l'affect n'est rien d'autre que la qualité sensible d'une relation. Précisons ce point en clarifiant les articulations entre relation, interaction et affect. Une relation est un rapport (ou un vecteur) intentionnel entre agents - et en ce sens, elle est plus qu'une simple relation logique. Si une relation est un rapport abstrait (virtuel), une interaction est toujours concrète (réelle) : elle est une relation actualisée hic et nunc par des acteurs singuliers, matérialisée dans des gestes, des paroles et des objets ${ }^{11}$. Enfin, l'affect est quant à lui sensible dans la mesure où il est toujours éprouvé par un individu dont il exprime le point de vue incarné ${ }^{12}$. Les affects sont ainsi les corrélats sensibles d'interactions concrètes, lesquelles actualisent des relations virtuelles ${ }^{13}$. L'affect est en quelque sorte le précipité sensible d'une relation. Il constitue par conséquent un indice relationnel particulièrement pertinent : il manifeste l'état, la teneur et la tonalité d'une relation, et cela aussi bien pour autrui (dont l'anthropologue) que pour soi-même. C'est donc à travers lui que les initiés éprouvent de manière tangible la réalité de la transformation initiatique. Derrière les affects qui s'expriment dans le rite initiatique, ou sont produits par lui, il ne faut en définitive chercher ni des sentiments privés (logique psychologique), ni des signes publics conventionnels (logique symbolique), mais d'abord des relations entre agents (logique relationnelle) ${ }^{14}$.

On connaît la polémique entre Lévi-Strauss et Turner ${ }^{15}$ autour de l'importance relative de l'affect et de l'intellect dans le rituel, le premier défendant une approche cognitive, le second une approche expérientielle. Plutôt que de me situer dans ce débat, je préfère opérer un déplacement du

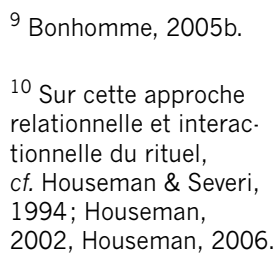

11 Je laisse en suspens l'épineuse question de la qualification

des interactions avec

des agents intentionnels non-humains (animaux, agents surnaturels, fétiches, etc.) ou des humains probléma tiques (doubles, âmes, ancêtres, etc.)

\section{Sur l'importance de l'« incarnation ou « incorporation» (embodiment) de l'expérience sociale, cf. Csordas, 1994.}

13 Réciproquement, l'expression des affects permet d'infléchir le

cours d'une interaction et, partant, de redéfinir la relation entre les acteurs.

Sur la conception interactionnelle-relationnelle des émotions,

cf. Bateson, 1991.

14 Pour cette raison, ce qui se passe au niveau des affects tient relativement peu à des facteurs socio. psychologiques. D'où le choix de la focale de la description ethnographique une mise au point sur le détail des interactions qui laisse en arrière. plan la sociologie du groupe et la psychologie des individus.

15 Lévi-Strauss, 1971 : 596.611; Turner,1969. 
${ }^{16}$ Bonhomme, 2005b.

17 Les termes vernacu. laires cités sont en getsogo, la langue des Mitsogo qui constitue le fonds commun de l'idiome rituel du bwete, y compris parmi les autres groupes ethnolin. guistiques.

Les autres termes locaux sont cités en « français du Gabon ». regard afin de mettre en perspective les deux approches. En effet, si le rituel induit des attitudes aussi bien affectives que cognitives, c'est qu'elles ne sont toutes deux que les propriétés émergentes d'un travail relationnel sous-jacent. J'ai analysé ailleurs le versant cognitif de ce travail relationnel en soulignant l'importance du secret, du mystère, du doute, du scepticisme et de l'ironie au sein du bwete misoko ${ }^{16}$. Le présent article s'intéresse à son versant affectif. Je me propose ainsi de montrer comment la dynamique relationnelle du processus initiatique se traduit - dramatiquement - par l'émergence d'affects spécifiques. Le parcours initiatique du bwete misoko étant long et complexe, je me limite cependant aux rites de passage (les visions d'eboga) et à la séance divinatoire préalable (la consultation du nganga). Je me propose de montrer comment la transformation dans l'interaction rituelle des relations asymétriques entre sorcier et victime, devin et patient, initié et initiateur permet la conversion d'un affect passif (les pleurs) en un affect actif (l'agressivité dirigée contre le sorcier). L'enjeu est finalement de mieux saisir, à partir d'un cas ethnographique singulier, les ressorts interactionnels et émotionnels de l'efficacité rituelle.

\section{En proie aux pleurs}

Le misoko est l'une des branches du bwete (ou bwiti), rite initiatique originaire des Mitsogo et des Gapinzi, populations du centre du Gabon (province de la Ngounié). Toutefois, même si ces derniers sont toujours considérés comme les experts traditionnels, le bwete s'est depuis longtemps répandu bien au-delà de sa contrée d'origine, dans la majeure partie du pays, des Bapunu au sud aux Fang au nord, des Myene sur la côte occidentale aux Bavove à l'est ${ }^{17}$. La branche mère du bwete, appelée disumba, est un rite de passage masculin : dans les villages et les lignages qui pratiquent le disumba, les jeunes hommes doivent se faire initier afin de faire pleinement partie de la communauté locale des hommes. Le bwete misoko forme quant à lui la branche thérapeutique du rite initiatique. Contrairement au disumba, l'initiation au misoko n'est pas obligatoire mais facultative et circonstancielle : un individu décide de s'y faire initier 
suite à l'épreuve accablante d'une infortune dont il suppose qu'un sorcier est le responsable. Les initiateurs, appelés nganga-a-misoko ou plus simplement nganga, sont aussi des devins-guérisseurs et soignent couramment des malades sans les initier. Ajoutons encore que si le disumba est strictement masculin, de plus en plus de communautés du misoko initient également les femmes (qui prennent alors le nom de mabundi).

Le bwete s'éparpille en une myriade de communautés locales implantées dans les villages ou les quartiers urbains autour d'un corps de garde (mbandja) dirigé par un père initiateur (nyima) dirigeant ses affaires rituelles de manière relativement autonome. Néanmoins, dans toutes les communautés, l'initiation au bwete impose l'ingestion des écorces râpées des racines de l'arbuste eboga (Tabernanthe iboga) aux propriétés hallucinogènes. Toute une nuit durant, le novice (banzi) doit avaler une dose massive de "bois sacré " et fixer attentivement un miroir dans lequel lui apparaissent des visions qu'il raconte au fur et à mesure à l'assemblée des initiés. Ce rite de passage visionnaire marque la nette séparation entre profanes et initiés : les premiers sont supposés ne rien savoir des secrets initiatiques puisque, contrairement aux derniers, ils n'ont pas "vu le bwete » de leurs propres yeux. Le contenu et la fonction de ces visions diffèrent toutefois selon les branches initiatiques. L'expérience visionnaire du disumba est un voyage au pays des ancêtres mythiques (le soleil Kombe, la lune Ngonde ou le premier ancêtre Nzambe-Kana), alors que celle du misoko concerne plus prosaiquement l'origine de l'infortune du banzi, origine qui est à chercher dans les agissements "mystiques" d'un parent malintentionné.

C'est ainsi une situation d'infortune attribuée à un sorcier (mogodo) qui conduit au bwete misoko. Ce fait jouant un rôle surdéterminant dans tout le processus initiatique, il convient dans un premier temps de clarifier la nature de cette affliction sorcellaire. Les classifications populaires opposent d'une part les "maladies naturelles " envoyées par Dieu (ebea-a-Nzambe) et d'autre part les « maladies mystiques ", causées par des esprits (ebea-a-migesi) ou des sorciers 
18 En raison de la matrilinéarité, l'oncle utérin est le premier suspecté (il est un sorcier quasi-légitime) Mais n'importe quel autre parent, tant du matri. que du patri-lignage, est possiblement un sorcier.

${ }^{19}$ Austen, 1993.

20 Pour des conceptions similaires au Cameroun, cf. Geschiere, 1995. (ebea-a-mogodo), c'est-à-dire dont l'origine est attribuée à un agent intentionnel malveillant, humain ou non-humain. Cette distinction étiologique ne se traduit pas dans une nosologie spécifique. En effet, une affliction causée par un sorcier repose généralement sur le cumul et la répétition de plusieurs registres du malheur: douleur chronique, blessures, incidents, misère, querelles familiales, décès de proches, etc. Cette répétition du malheur fait prendre conscience que quelque chose ne va pas. Mais ce quelque chose se soustrait à l'appréhension, planant au-dessus de l'existence comme une menace imprécise mais omniprésente : «J'avais l'impression que quelque chose n'allait pas dans ma vie, mais je ne pouvais pas vraiment définir ce que c'était", selon le témoignage d'un malade. L'angoisse - qui se manifeste notamment dans des cauchemars obsédants - constitue ainsi une dimension essentielle du malheur. Cette détresse aiguë s'accompagne du sentiment d'être enfermé durablement dans l'infortune, mais aussi d'être injustement dépossédé. "Mes chances sont bloquées " est ainsi une expression récurrente - le terme " chance " (ou parfois " étoile ") désignant le capital de réussite d'un individu, la capacité personnelle à mener à bien ses désirs et ses projets, à réussir sa vie.

Quelqu'un est à l'origine de cette situation d'infortune. La répétition du malheur amène en effet inévitablement à suspecter l'existence d'un responsable. La sorcellerie opérant le plus souvent à l'intérieur de la parentèle, et même du lignage, c'est parmi ses propres parents qu'il faut chercher le coupable ${ }^{18}$. En effet, la dynamique du pouvoir et de la fortune au sein du lignage est un jeu à somme nulle: chaque gain est compensé par une perte, chaque gagnant par un perdant. L'ambitieux doit nécessairement "donner " (c'est-à-dire sacrifier) un parent pour s'approprier son pouvoir et monopoliser les " chances " du lignage. Cette "économie morale ${ }^{19}$ " fait de la sorcellerie un mal nécessaire sur le chemin du succès ${ }^{20}$. Si la solidarité lignagère est sans cesse réaffirmée en public, elle est en réalité constamment menacée par l'ambition personnelle, l'envie et la sorcellerie. Lorsqu'un individu se trouve en proie au 
malheur, les ragots qui circulent inéluctablement dans la famille cristallisent alors bien vite en une imputation de sorcellerie. Toutefois, ce ne sont là que des suspicions, la victime ne pouvant jamais être certaine de l'identité de son persécuteur. Dissimulation, incertitude et opacité sont en effet les caractéristiques premières de la sorcellerie : il n'est pas rare que le sorcier répande lui-même des rumeurs pour se disculper, ou bien qu'il revête le masque d'un parent innocent afin de le faire accuser à sa place. On ne peut voir la sorcellerie, on en subit seulement les effets.

Cette agression sorcellaire est le plus souvent pensée en termes de prédation cannibale. Le sorcier est en effet couramment associé à la panthère, prédateur carnivore par excellence. Il «bouffe » sa victime qui est pour lui une "viande" (nyama), c'est-à-dire un gibier, une proie. C'est ce qu'on appelle "sortir en vampire" (vembaga) : l'esprit du sorcier quitte nuitamment son corps pour se rendre au chevet de sa victime endormie et dévorer sa force vitale à son profit. Cette perte de substance vitale se traduit chez la victime par une faiblesse chronique et par un sentiment angoissé d'impuissance et d'enfermement dans la répétition du malheur. L'infortuné n'a plus aucune prise sur le cours de son existence et se sent en permanence entravé. Il est une proie passive incapable de réagir face à son persécuteur. Ces images prégnantes de la dévoration de la force vitale et du blocage des chances traduisent ainsi l'expérience de la passivité au principe de l'affliction. Puisque la force vitale et la chance désignent la puissance d'agir de tout individu, l'agression sorcellaire consiste en une dépossession de la puissance d'agir de la victime au profit égoïste du sorcier.

En définitive, la sorcellerie repose sur une relation asymétrique entre le sorcier et sa victime qui se déploie sur fond d'infortune ${ }^{21}$ : relation de perception (voir sans être vu versus être vu sans voir) et de prédation (prédateur tout-puissant versus proie passive). La sorcellerie est donc une relation virtuelle plutôt qu'une interaction concrète, puisque par définition le sorcier reste invisible. Et cette impuissance de la victime à interagir avec son persécuteur constitue justement un aspect central de l'expérience 
22 Sur les contextes relationnels de l'agentivité sociale, cf. Gell, 1998. affective de l'affliction : elle pâtit au sens où elle subit sans pouvoir agir en retour. La sorcellerie implique ainsi une conception fondamentalement relationnelle de l'agentivité humaine $\left(\right.$ agency $\left.^{22}\right)$. Le recours au bwete misoko ne peut être compris hors de ce contexte, puisque tout le processus initiatique vise justement à saper cette relation sorcellaire afin de redevenir actif. Ce n'est cependant qu'au terme d'une consultation chez un devin (également initiateur) que la victime décide de se faire initier. Épisode déterminant, cette séance divinatoire constitue ainsi le point d'entrée dans le processus initiatique.

Un patient décide de consulter un devin-guérisseur (nganga-a-misoko - du verbe sokogo, " découvrir ") afin de préciser l'origine de son infortune, de conforter ses soupçons et de se voir ainsi confirmer dans le statut de victime. Cette séance divinatoire a lieu soit lors d'une veillée de bwete, soit plus simplement pendant la journée à la demande expresse $\mathrm{du}$ "malade " (mobea). Le patient commence par tendre au nganga les articles rituels (bougie, aiguille, boisson) et sa contribution financière (quelques milliers de francs CFA). Il doit ensuite donner à haute voix son nom, puis ceux de ses deux parents. Ces trois noms constituent la carte d'identité lignagère du patient : un individu singulier rattaché par le biais de ses parents à deux lignages distincts (« le côté des mères ", "le côté des pères "). Cette nomination est importante dans la mesure où la consultation consiste avant tout à resituer l'infortune dans le champ de la parentèle du patient, en évaluant les responsabilités respectives des deux lignages. Le malade se présente ensuite de face, puis de dos au regard examinateur du devin. À la lumière de sa torche, ce dernier scrute alternativement le patient et son petit miroir divinatoire. Il trace alors une série de signes au kaolin sur le corps du patient. À chaque partie du corps correspond analogiquement un trouble, une prémonition ou un traitement : par exemple, une marque au pied désigne un "fusil nocturne " (bota-a-pitsi - agression sorcellaire qui touche habituellement le pied), un trait à l'épaule annonce une promotion (recevoir un "galon»), un trait du sternum à la bouche prescrit un vomitif. Le principal schéma organi- 
sateur de ce code divinatoire est la symétrie droite-gauche, qui correspond à l'opposition entre patri- et matri-lignage. Un trait le long du bras droit désigne ainsi le patrilignage, le long du bras gauche le matrilignage. Si ce trait est coupé perpendiculairement au niveau du poignet, cela signifie que le lignage concerné porte une part de responsabilité dans l'infortune; s'il n'est pas coupé, qu'il est innocent. En donnant à voir l'infortune à la surface du corps du patient, ces signes divinatoires permettent en définitive d'extérioriser son malheur intime et de rendre visible les agressions invisibles du sorcier.

Le devin passe ensuite à la partie orale de sa consultation, brodant autour de l'interprétation des signes au kaolin. Il énonce un certain nombre de propositions miassertives mi-interrogatives concernant la vie du patient, les maux dont il souffre et leur origine. Il saute d'un registre du malheur à l'autre, suggérant entre eux des connexions non fortuites afin de dessiner l'image d'une infortune massive qui afflige toute la vie du malade ${ }^{23}$. Nombre des révélations concernent l'étiologie sorcellaire de l'infortune, confirmant ainsi les soupçons du patient. Il est cependant rare que le nganga accuse nommément quelqu'un. Il se contente habituellement de peser les responsabilités respectives des matri- et patri-lignages en des termes généraux et ambigus. La consultation s'achève par la proposition d'un traitement, ou d'une initiation si le cas est jugé trop préoccupant ou obscur. L'initiation au bwete misoko est ainsi présentée comme la possibilité pour le malade d'aller voir par luimême l'origine de son mal, d'en identifier le coupable et d'y trouver un remède.

La portée de cette séance divinatoire est moins une simple affaire d'adéquation (entre des états de fait et les énoncés du devin) que de pertinence relationnelle et affective. Le patient se sent enfermé dans un état de perplexité impuissante qui le rend incapable d'articuler de manière cohérente son propre malheur. La consultation insère alors cette expérience affective dans un cadre interactionnel singulier. Le patient écoute en silence le devin qui lui parle de son infortune. Il doit se contenter d'acquiescer 
24 L'assistance joue également un rôle dans cette objectivation du malheur du patient : ce sont généralement les autres initiés présents

mais aussi les quelques parents du patient assistant à la consulta tion qui confirment les propos du devin par les «Basé! » les plus appuyés. Sur le rôle décisif des tiers (annonciateurs ou confirmateurs) dans les affaires de sorcellerie, cf. Favret-Saada, 1977. par la formule rituelle «Basé! » si les révélations du devin lui semblent justes, sans ajouter aucune autre précision. S'il veut trop en dire, il se fait aussitôt réprimander par le nganga: "Ce n'est pas le malade qui consulte. " Cette admonestation rappelle que la consultation est bien une divination. Il pourrait sembler beaucoup plus simple que le patient expose par lui-même les maux qui l'amènent chez le nganga afin que ce dernier puisse les soigner. Il est pourtant crucial que ce soit le nganga - à la fois devin et guérisseur - qui révèle au patient ses symptômes et la nature de son affliction. Ce que le malade ne peut formuler à propos de lui-même, le devin le lui dit, l'exprime à sa place. Son expérience du malheur - vécu intime dont le devin devrait pourtant tout ignorer - se trouve brutalement objectivée, saisie, attrapée dans le discours d'un tiers ${ }^{24}$. L'effet produit est violent. Au début de la séance, le malaise des patients est palpable. Leurs postures corporelles sont embarrassées, tandis que le nganga les marque de signes au kaolin. Ils répriment avec difficulté des rires de gêne et paraissent décontenancés. Mais vers la fin de la consultation orale, les patients éclatent bien souvent en sanglots. De leurs propres points de vue, ces pleurs trahissent le sentiment troublant d'avoir été " percé à jour " par le nganga : " vous avez touché ce qui est au fond de moi ", comme l'avouait un patient en larmes au terme de la séance divinatoire. Alors que l'embarras initial traduit plutôt une certaine incertitude du patient concernant l'interaction divinatoire, les pleurs conclusifs sont reconnus par les devins comme la plus belle preuve du succès de leur consultation.

Cette irruption de l'affect dans la séance divinatoire obéit à une logique relationnelle. Les sanglots du patient reflètent sa propre position dans la relation divinatoire, position que l'on peut qualifier de passive par rapport à celle du devin. Le patient est vu, le devin voit. Le patient ignore, le devin sait. Le patient reste immobile, le devin dessine sur son corps. Le patient se tait, le devin parle. Si les pleurs sont un substitut affectif pour exprimer le malheur quand les mots manquent, ils sont aussi un effet direct de la parole du devin qui, lui, sait trouver ces mots. Le malade 
se trouve ainsi piégé par le devin dans la consultation, de même que le "film " de son existence est prétendument capturé dans le miroir divinatoire. Cette métaphore du piégeage est appropriée : les nganga décrivent en effet leur consultation comme un piège de chasse (getambo) tendu pour attraper un malade qui est, lui, comparé à un gibier (nyama). Les signes au kaolin, le miroir et les révélations orales sont autant de ruses rituelles permettant de piéger le malade dans les rets de la relation divinatoire. Cette image de la proie prise au piège souligne bien la position passive du patient dans la relation divinatoire. Et les pleurs sont la traduction affective de ce saisissement.

Cette passivité du patient dans la relation divinatoire vient redoubler sa passivité en tant que victime dans la relation sorcellaire. D'une part, la séance divinatoire confirme que son affliction est liée à une situation sorcellaire dans laquelle il est tenu prisonnier. D'autre part, la même image de la proie est employée pour exprimer la position passive dans les deux relations. Certes, les prédations sorcellaire et divinatoire diffèrent l'une de l'autre. D'un côté, on a une dévoration cannibale perpétrée par un sorcier comparé à une panthère sanguinaire. Cette prédation sorcellaire est agressive et malintentionnée. De l'autre, on a une capture opérée par un devin comparé à un chasseur-piégeur rusé mais bienveillant. Le piégeage renvoie ainsi à l'intelligence de l'artifice technique, intelligence qui se retrouve dans la ruse rituelle. Mais par-delà ces différences, le patient se trouve bien dans la position de la proie tant vis-à-vis du sorcier que du devin : il est doublement " pris ". La relation divinatoire n'opère pas pour autant un simple redoublement de la relation sorcellaire. L'actualisation de la relation sorcellaire virtuelle dans une interaction divinatoire concrète permet en effet de l'infléchir sensiblement. La sorcellerie est une relation problématique : elle repose au départ sur des soupçons à l'égard d'un sorcier mal identifié car invisible. La divination introduit un tiers personnage (devin) occupant une position en surplomb qui permet une mise en perspective de la relation duelle entre la victime et le sorcier. À travers un jeu de signes et d'énoncés, le devin 
${ }^{25}$ Ces pleurs ne sont donc pas réductibles à un signe conventionnel, comme cela pourrait être le cas pour les larmes lors de rites funéraires. Le patient n'est ici pas tenu de pleurer, et souvent il ne pleure pas du tout. Ses pleurs émergent comme l'effet

incontrôlable d'une interaction divinatoire réussie : ils ne symbol isent pas la relation, ils l'actualisent.

${ }^{26}$ Houseman, 2003.

${ }^{27}$ Bateson, 1977. confère alors une certaine forme de certitude et d'évidence à une relation virtuelle jusque-là très incertaine. Les sanglots constituent en définitive une cristallisation affective liée à l'actualisation sensible de la position passive du patient dans la relation sorcellaire par l'intermédiaire de l'interaction divinatoire ${ }^{25}$ [voir figure 1 ci-dessous].

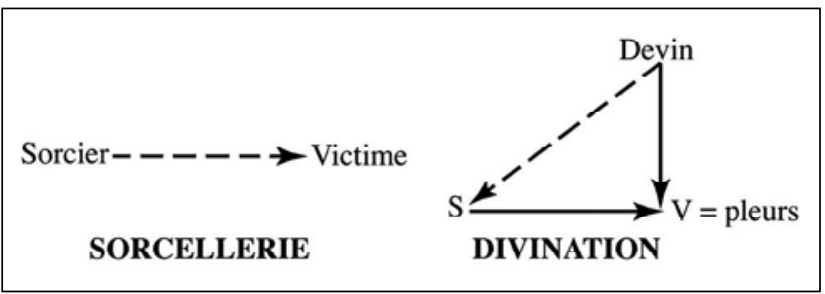

Figure 1 - Relation sorcellaire et relation divinatoire

\section{« Taper le serpent ॥}

L'irruption des pleurs peut donc être comprise comme un effet sensible lié à la position spécifique occupée par un individu dans un champ de relations asymétriques. Du point de vue du malade, la qualité ou tonalité dominante de ces deux relations est bien la passivité. On pourrait pourtant dire que la décision d'aller consulter un devin constitue déjà une reprise d'initiative de la part du patient qui désire activement sortir de l'infortune. C'est cependant en réalité souvent son entourage qui incite, et parfois même oblige, un individu à aller chez un devin. On s'adresse donc à un nganga lorsque cela ne va vraiment plus et que l'on se sent dépassé. La possibilité d'une reprise d'initiative semble donc passer par la reconnaissance de son impuissance à changer la situation par soi-même. Le recours au devin est en cela comparable à la décision d'entamer une psychothérapie ${ }^{26}$ ou encore au scénario de guérison au sein des Alcooliques Anonymes $^{27}$ : "toucher le fond" est en effet pour l'alcoolique la condition sine qua non de l'enclenchement du processus thérapeutique. Seule une passivité maximale peut ainsi entraîner une reprise d'initiative. Or, les sanglots du 
patient indiquent ce point où il touche le fond - tout l'art du devin étant justement de provoquer cette brutale prise de conscience. C'est pourquoi si les pleurs sont un affect passif sans réelle valeur cathartique, ils sont cependant aussi un affect réflexif : pleurer constitue un retour sur soi. La séance divinatoire consiste en effet à faire saillir la relation sorcellaire au premier plan de l'expérience du patient. Cette relation sorcellaire émerge ainsi comme un enjeu explicite pour le patient, même s'il n'a pas encore de réelle prise sur elle puisqu'il reste dans la position passive de la proie. Sur fond de cette prise de conscience réflexive, l'étape suivante $\mathrm{du}$ processus initiatique - le rite de passage proprement dit - opère alors une véritable inversion relationnelle ${ }^{28}$. Logique divinatoire et logique initiatique se répondent en effet directement. La transformation initiatique passe ainsi par une transformation conjointe des relations et des affects : l'initiation est à la fois une auto-divination et une inversion de la relation sorcellaire; en conséquence, les pleurs sont désormais bannis et doivent laisser place à des affects plus actifs, ce qui s'exprime notamment dans la riposte agressive contre le sorcier.

Les rites de passage du bwete misoko comprennent deux phases distinctes. En forêt, pendant la journée, une série de rituels purificateurs et prophylactiques doit garantir que le voyage visionnaire du néophyte (banzi) se passera sans encombre. Pendant ces préliminaires, le banzi reste dans une position passive : il est en effet soumis à des manipulations corporelles (fumigation, bain, onction, scarification), sans aucune initiative possible de sa part, ni réelle compréhension de ce qu'on lui fait subir. De retour au village à la tombée du jour, les initiateurs installent alors l'initié au mbandja, le corps de garde où ont lieu toutes les veillées de $b_{w e t e}{ }^{29}$. C'est là que le banzi, assis sur une natte face à un miroir, va manger les racines hallucinogènes d'eboga toute la nuit durant afin d'avoir des visions ${ }^{30}$. L'initié doit fixer attentivement son propre reflet dans le miroir, sans détourner le regard ni ciller trop souvent sous peine de rappel à l'ordre. L'initiation constitue en effet un retour sur soi : lorsqu'il focalise son attention sur le miroir, l'initié se con-

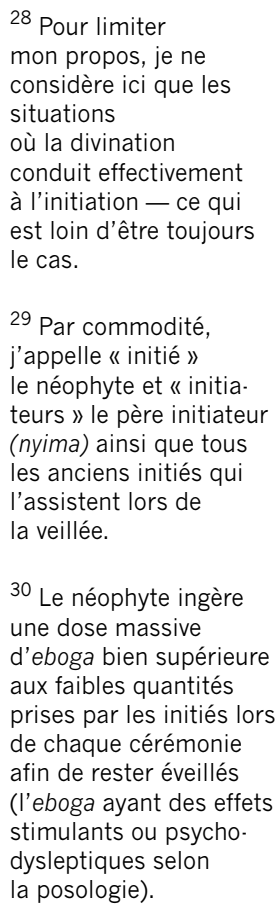


31 Toutes les visions citées proviennent de différents récits vision. naires que j'ai recueillis pendant la nuit même de l'initiation, au fur et à mesure que les banzi les racontaient à l'assistance.

32 Le patient peut en effet toujours suspecter que les étranges révéla. tions du devin sont des mensonges, ce dernier jouant explicitement sur ce point pour induire chez son patient autant la perplexité que

la curiosité. centre sur lui-même et sur son malheur. Et quand les effets de l'eboga commencent à se faire sentir, avant toute autre vision, le banzi observe habituellement une métamorphose de sa propre figure : " je me transforme en gorille», « je suis comme un Pygmée ", "j'ai le visage d'un vieillard " ${ }^{31}$. Ces transformations spéculaires font de l'initiation une véritable auto-divination. Si le nganga voit la vie du patient dans son petit miroir divinatoire, c'est maintenant le banzi qui peut découvrir de ses propres yeux l'origine et les remèdes possibles de son infortune dans le grand miroir initiatique. Et le devin propose d'ailleurs l'initiation à son patient comme une promesse de vérification personnelle de la consultation : puisque " tous les nganga sont des menteurs ${ }^{32}$ ", il vaut mieux que le malade aille par lui-même « voir comment est son corps » dans le miroir. Selon l'expression récurrente, " il faut aller voir pour le croire ", c'est-à-dire qu'il faut voir en personne dans l'initiation pour croire à cette parole rapportée qu'est la consultation.

Cette réflexivité spéculaire ne signifie pas pour autant que l'expérience visionnaire soit solipsiste. Elle est au contraire un processus collectif auquel l'assistance des initiateurs participe autant que l'initié. Les initiateurs demeurent en effet en permanence aux côtés de l'initié qui doit leur raconter toutes ses visions au fur et à mesure qu'elles lui apparaissent. Chaque récit est alors ponctué par les «Basé! " enthousiastes des initiateurs qui valident de la sorte les visions de l'initié. Il s'agit là d'une inversion par rapport à la séance divinatoire : ce n'est plus le patient qui acquiesce aux révélations du devin, ce sont maintenant les initiateurs qui acquiescent aux révélations de l'initié. Les «Basé!» de l'initiation sont un écho inversé des «Basé! » de la divination. Les deux configurations relationnelles se trouvent donc dans un rapport de symétrie inversée, les positions des acteurs pivotant autour du miroir divinatoire. Dans le premier cas, le devin voit dans le miroir et parle, tandis que le patient acquiesce. Dans le second cas, le patient devenu initié voit dans le miroir et parle, tandis que les initiateurs - qui étaient auparavant les devins - acquiescent. La relation initiatique constitue en définitive une inversion de la 
relation divinatoire [voir figure 2 ci-dessous] - ce qui atteste que l'initiation est une auto-divination, une vérification personnelle de la consultation.

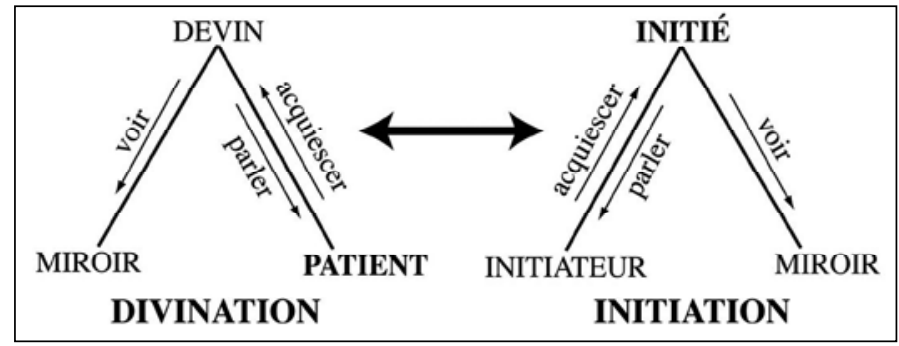

Figure 2 - Relation divinatoire et relation initiatique

Le temps de la veillée, l'initié se trouve ainsi dans la position du devin. Il ne se contente d'ailleurs pas de voir sa propre vie dans le miroir. Il peut encore y voir celle des autres, ce qui lui donne le pouvoir de se livrer à une véritable consultation divinatoire. D'où le fait que les visions de l'initié ne lui appartiennent pas en propre et doivent être racontées aux initiateurs. L'assistance, impatiente d'entendre ces précieuses révélations, réclame généralement d'elle-même la consultation : "Dis-moi un peu comment tu vois mon corps!»; "Le bwete ne te montre pas des choses sur moi? "Au cours d'une initiation, une banzi révèle ainsi au père initiateur les raisons de ses problèmes financiers : «La main de Papa est percée, c'est pourquoi il ne peut pas garder de l'argent. » Plus tard, la même initiée, très exaltée, s'adresse à une parente venue assister à la veillée : elle lui donne une bénédiction, front contre front, puis lui fait des révélations encourageantes sur son couple, ce qui fait immédiatement éclater en sanglots ladite parente. Cette situation est révélatrice : alors que le patient se mettait à pleurer lors de la consultation, c'est maintenant lui qui fait pleurer les autres. Il quitte ainsi la position passive du gibier pris au piège du devin pour venir occuper la position active du piégeur, en étant à son tour capable de capturer autrui dans le jeu rituel du miroir et de la consultation. Du point de vue affectif, cela s'accompagne habituellement d'une 
33 Sur la frayeur

dans les visions,

cf. Deshayes, 2002. véritable jubilation de la part de l'initié qui tire un plaisir visible de la situation. Le pouvoir divinatoire de l'initié permet en effet une véritable reprise d'initiative : le banzi peut prendre publiquement la parole. Et cette parole est une parole efficace, prise au sérieux, ce qu'il peut constater dans ses effets les plus sensibles : l'approbation enthousiaste des initiateurs ou les pleurs d'autrui.

Par le biais d'une permutation actantielle, l'initiation constitue ainsi une inversion de la relation divinatoire : le patient est temporairement devin de soi et d'autrui. Mais la transformation initiatique touche également la relation sorcellaire, ce que montre bien le détail des visions initiatiques. Dans le bwete disumba, tous les banzi sont censés voir les mêmes visions stéréotypées, les cadets effectuant le même périple visionnaire que leurs aînés. Cette vérification personnelle de la mythologie collective doit insuffler aux jeunes hommes le sentiment d'appartenir à une communauté masculine dont l'identité immuable a été léguée par les ancêtres. À l'opposé, le bwete misoko se caractérise par une individualisation des visions, puisque chaque banzi vient y découvrir l'origine de son infortune personnelle. Cependant, la comparaison de différents récits visionnaires du misoko révèle de nombreux traits récurrents et finalement une remarquable uniformité. Il est par conséquent possible de dégager un scénario idéal-typique des visions du bwete misoko.

Les premières visions de la nuit ne sont généralement que de brèves apparitions se succédant de manière incohérente : visages, parents ou inconnus, animaux, objets, paysages que l'initié observe comme un spectateur extérieur. Ce bombardement d'images (souvent appelées "flashs") s'accompagne habituellement du surgissement d'affects très labiles : frayeur, perplexité, désarroi, brusque oscillation du rire aux larmes ${ }^{33}$. Flashs et frayeurs manifestent que le banzi se trouve encore dans une position passive, subissant les visions. Mais dans d'autres visions plus élaborées (appelées «films »), l'initié parvient progressivement à sortir de cette perspective passive pour acquérir un regard omniscient, 
qui lui donne la capacité de déceler ce qui échappe à la perception ordinaire. Cette perception omnisciente est directement liée à la sorcellerie. L'initié parvient à observer les agissements du sorcier qui manigance, pensant ne pas pouvoir être observé (scènes de décèlement) : « je vois ma première femme cuisiner un poisson et prélever l'arête pour fabriquer un poison "; « la grand-mère des enfants de ma grande sœur a un bâton de la nuit [sexe "mystique"]. Elle entre dans la chambre de ses petits-enfants pour les séduire avec ce bâton ". L'initié parvient également à voir son propre corps pris dans l'affliction sorcellaire (scènes autoscopiques) : un homme observe, comme à distance, son pied attaché par des feuilles; une femme voit sa propre tête, coupée et posée sur des braises. Il arrive encore à détecter les objets maléfiques dissimulés chez lui par le sorcier (scènes diascopiques) : «Au niveau de la porte de la cuisine se trouve un serpent ["mystique"]. C'est là où le sorcier urine. Derrière la porte de la cuisine, il y a un trou à l'intérieur duquel l'ex-femme de tonton K. met le poison. »

Les initiés répètent avec insistance que le bwete permet de "voir l'invisible " et d'accéder à un "monde mystique ". Pourtant, le visionnaire ne découvre pas un autre monde, surnaturel et fantastique. Ses visions sont plutôt des scènes de la vie quotidienne, et même souvent des scènes de la vie familiale. Les protagonistes principaux font généralement partie de sa parentèle. Mais les visions concernent le revers nocturne de la parenté : ce que les parents font en plein jour laisse la place à ce que ces mêmes parents, poussés par la jalousie, font la nuit et dans le dos des autres. Le monde invisible du bwete misoko n'est donc rien d'autre que le monde invisible de la sorcellerie, vu sous un autre éclairage et un autre point de vue ${ }^{34}$. Voir l'invisible signifie percevoir les actes occultes au principe de la sorcellerie, être le témoin paradoxal de scènes sans témoins. L'initiation donne l'occasion inestimable - car normalement impossible - de prendre le sorcier en flagrant délit. Ces scènes de décèlement constituent par conséquent un épisode central du scénario visionnaire.

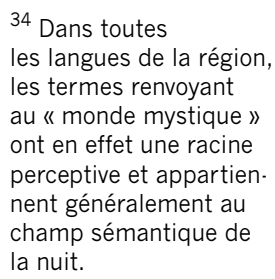


${ }^{35}$ Au cours de son voyage visionnaire, le banzi doit impéra. tivement recevoir d'un tiers son kombo (ainsi que sa signification ésotérique), nom initia. tique qui remplacera désormais son nom civil dans toutes les interactions avec les initiés, marquant bien que l'initiation est une seconde naissance.

${ }^{36}$ Certes, une « interac. tion imaginaire » est un oxymore, dans la mesure où l'interaction a été définie comme une relation concrète, réelle. Mais l'imaginaire renvoie ici moins à l'irréel qu'au pouvoir (proprement stupéfiant) des images.
Dans d'autres visions, l'initié est le témoin de scènes qui s'adressent directement à lui. Dès lors qu'il est pris dans le point de vue d'un tiers (même imaginaire), il ne peut plus maintenir sa situation de spectateur extérieur et passe lui-même dans l'image, comme s'il était happé par le miroir. Les interactions deviennent alors possibles. Les personnages rencontrés se répartissent en deux catégories selon la relation qu'ils entretiennent avec l'initié : les adjuvants (parents bienveillants, père initiateur, sirènes, Pygmées et, bien souvent, des Blancs) et les opposants (parents sorciers et leurs complices, serpents, fantômes et autres mauvais esprits). Les interactions avec les adjuvants sont habituellement des dons (plantes médicinales, ritualia, argent, marchandises), des révélations de secrets (nom initiatique, secrets familiaux, secrets rituels, chansons) ou des bénédictions $^{35}$ : «dans le hall d'une grande maison, des Blancs me remettent des clés et me disent que la maison m'appartient "; « mon père me dit que son cœur et son crâne sont pour moi ». Ces rencontres sont des anticipations du salut et des promesses de fortune.

À l'opposé, les interactions avec les opposants consistent en une série d'agressions et de ripostes. À travers ses visions, l'initié est donc en interaction avec le sorcier puisque ce dernier peut toujours réagir. Les deux protagonistes ont en effet le pouvoir de modifier le cours de l'interaction - d'où le fait que l'aventure visionnaire ait toujours une issue imprévisible. Le miroir et l'eboga permettent ainsi d'actualiser une relation d'abord seulement virtuelle (le sorcier étant toujours absent) dans une interaction imaginaire ${ }^{36}$. Dans le combat entre l'initié et son sorcier, la perception visuelle est souvent mise en scène, devenant l'enjeu principal de la vision. Cette mise en abyme de la perception concerne les innombrables scènes de croisement de regard, d'œillade furtive ou de dissimulation: "une femme me tourne le dos lorsque je la regarde ", "ma mère en train de me lorgner ", "ma mère se cache le visage avec une planche ». Ces scènes illustrent le rôle décisif de la perception dans la relation sorcellaire. Les personnages masqués qui hantent les visions relèvent du même registre perceptif: 
"un personnage masqué qui cache une parente»; «un homme qui porte des lunettes noires. Il me dit qu'il est là pour espionner. Lorsqu'il retire ses lunettes, je reconnais mon oncle ». Le masque est en effet le visage préféré du sorcier, la sorcellerie étant par définition une dissimulation.

Ces mises en scène de la perception ressemblent ainsi à un jeu de cache-cache à l'issue incertaine entre l'initié et le sorcier, chacun cherchant à voir l'autre sans être vu par lui. L'enchaînement des visions de S. D. (un jeune homme) illustre fort bien l'infléchissement de la relation sorcellaire au cours de la nuit (j'omets un certain nombre de visions intercalées). Scène 1 (parmi les premières de la nuit) : « Ma figure n'arrête pas de changer : je me transforme en gorille puis en femme. Mais ce n'est pas vraiment ma figure. Derrière ces films, quelqu'un se cache que je n'arrive pas encore à identifier. "Scène 2 : «Un personnage masqué dont je n'arrive pas à découvrir le visage. "Scène 3: "Je sens une présence derrière moi. " Lorsque $\mathrm{S}$. D. assène (réellement) des coups de chasse-mouches, cette présence disparait. Scène 4 : le père initiateur passe (réellement) sa torche derrière S. D.; ce dernier aperçoit alors une personne dont il n'arrive pas à identifier le visage. Scène 5 : "Tonton K. est au village. Il est bien habillé. Il se cache en plusieurs endroits et porte plusieurs masques. Je le poursuis jusqu'au fond d'une grotte dans une forêt. Je lui ôte finalement son masque et découvre sa figure. "Scène 6 : «Je suis enchaîné dans une rivière, avec un seul bras libre. Je bouge : la chaîne se coupe et disparaît." Scène 7 : "Le visage de tonton K. n'arrête pas de se transformer, tantôt noir tantôt blanc. Il se cache derrière moi. " Scène 8 (l'une des dernières de la nuit) : "Mon oncle se retrouve là où j'étais attaché dans la rivière, ligoté par des chaînes."

Les scènes 1, 2, 4 et 7 manifestent l'impuissance de l'initié qui perçoit le sorcier mais n'arrive pas à l'identifier à cause de ses masques. Comble du travestissement sorcellaire, dans la première scène, le sorcier va jusqu'à prendre l'apparence de l'initié, sa propre victime, pour ne pas être reconnu. Ces visions induisent chez S. D. un désarroi palpable. Les coups de chasse-mouches dans la scène 3 
constituent la première réaction active. $\mathrm{Si} \mathrm{S}$. D. n'arrive pas encore à identifier son persécuteur, il parvient au moins à le repousser. Fait révélateur, les coups sont bien réels : c'est S. D. assis face au miroir, et pas seulement son double imaginaire dans le miroir, qui assène des coups avec le chassemouches qu'il doit brandir tout au long de la nuit. Ces mimes de combat, fréquents au cours des initiations, permettent ainsi aux banzi de véritablement agir leurs visions, ajoutant le poids d'un acte moteur réel à la riposte imaginaire. La scène 5 est décisive : S. D. parvient à identifier son oncle utérin en le poursuivant puis le démasquant. La joie de S. D. se réverbère alors dans les applaudissements de l'assistance enthousiaste. La sorcellerie étant une dissimulation occulte, découvrir le visage du sorcier signifie en effet le faire brutalement sortir de l'invisible. L'identification visionnaire du sorcier est un épisode-clé du scénario visionnaire, même si la plupart du temps l'initié suspectait déjà l'identité de son persécuteur. La vérification perceptive en première personne contraste avec les suspicions initiales qui n'étaient fondées que sur des présomptions incertaines ou des ragots colportés par des tiers. La scène 6 représente un nouvel état d'impuissance : ligoté dans une liane, S. D. est victime d'une "corde", terme commun qui désigne le serpent mbumba, auxiliaire maléfique du sorcier dont l'action constrictrice est une métaphore transparente du blocage et de l'emprisonnement dans l'infortune. La délivrance visionnaire de l'initié représente alors une auto- "coupure de corde ", rituel opéré par les nganga pour libérer un malade de l'emprise d'un mbumba. La traque de la scène 5 s'achève dans la scène 8 qui opère un retournement complet de la relation sorcellaire : l'oncle sorcier se retrouve enchaîné à la place de l'initié. C'est ce que les nganga appellent communément un "retour à l'envoyeur " : l'arme du sorcier est retournée contre lui. S. D. jubile.

Il y a ainsi une véritable téléologie visionnaire. L'initié commence par assister à des scènes en simple spectateur passif. Il devient progressivement partie prenante des visions pour finalement y tenir le rôle de l'acteur principal. Ce processus d'activation du rôle de l'initié dans ses propres 
visions passe idéalement par une série de péripéties décisives que l'on retrouve dans la plupart des scénarii visionnaires [voir figure 3 ci-dessous] : scène de flagrant délit (déceler la sorcellerie), scène d'identification (démasquer le sorcier), scène de riposte (attaquer le sorcier, détruire ses fétiches maléfiques), scène de délivrance (se libérer de l'affliction).

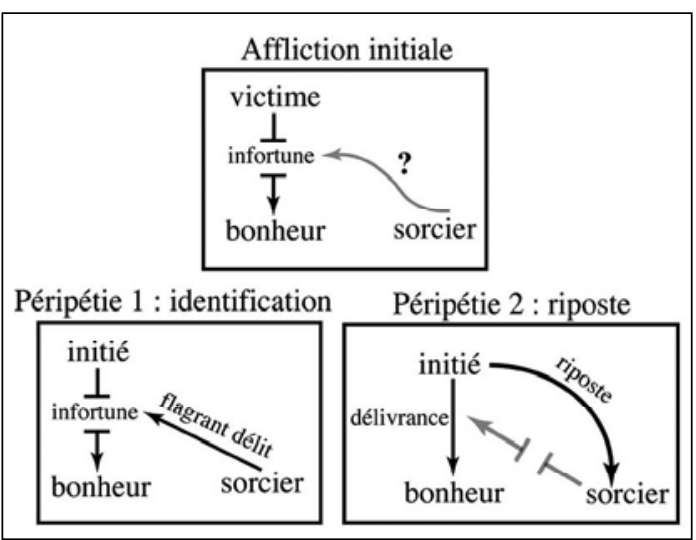

Figure 3 - Inversion visionnaire de la relation sorcellaire

Le retournement de la relation sorcellaire oriente tout le scénario visionnaire. L'initiation ne vise donc pas la réconciliation entre parents brouillés. Elle n'abolit pas la relation sorcellaire, mais la prolonge en la transformant. Entre les premières visions au début de la nuit et les dernières le lendemain matin, les pôles de la relation ont tendance à s'inverser. La victime est initialement dans la position d'une proie impuissante à la merci d'un prédateur invisible. Mais l'initié parvient progressivement à saper la double relation asymétrique de perception et de prédation qui l'unit malgré lui au sorcier, allant même jusqu'à la retourner à son avantage. C'est alors le sorcier qui se retrouve en position de proie traquée par un prédateur omniscient et omnipotent: "Mon grand frère [l'un des sorciers] vient me dire : "comment fais-tu pour nous voir alors que nous ne te voyons plus? Je t'ai cherché partout, mais je n'arrivais pas à te trouver!" " Ce retournement serait toutefois impossible sans l'aide des adjuvants, notamment les parents 
37 Je laisse en suspens l'épineuse question de l'effectivité de cette reconfiguration relation. nelle virtuelle. La portée et la temporalité du processus initiatique dépassent bien entendu le cadre rituel. Mais comment le travail rela. tionnel accompli dans les visions initiatiques se traduit-il dans les interactions sociales ordinaires (entre l'initié et son parent sorcier notamment)?

38 Sur la construction collective d'une réalité invisible dans

un contexte rituel, cf. Schieffelin, 1985 ; Taylor, 1993. bienveillants dont l'initié cherche à obtenir la bénédiction dans ses visions. Le combat du banzi contre son sorcier ne peut l'amener à se couper totalement de sa parentèle pour revendiquer une pleine autonomie personnelle : une telle situation équivaudrait à une extrême faiblesse et engendrerait donc une vulnérabilité encore accrue à la sorcellerie. L'initiation visionnaire opère en définitive un véritable travail de reconfiguration du réseau relationnel - notamment celui de la parentèle - dans lequel l'initié est virtuellement pris et sur fond duquel son malheur s'inscrit ${ }^{37}$.

Toutes les interventions des initiateurs au cours de la veillée visent précisément à faciliter ce retournement de la relation sorcellaire. Les initiateurs guident en effet l'initié tout au long de la nuit, jouant ainsi un rôle actif dans le façonnage des visions. Ils pilotent l'initié par un jeu insistant de questions et de directives, comme un metteur en scène faisant de la direction d'acteur. Ces directives sont typiquement des injonctions d'actions: les initiateurs incitent l'initié à s'impliquer dans ses propres visions, à dépasser le point de vue du spectateur pour devenir acteur. Par exemple, au récit de la vision d'un personnage, les ordres fusent pour que l'initié lui parle ou exige de lui un présent. Si ce personnage se dirige quelque part, l'initié doit le suivre. S'il a l'air menaçant, il doit le frapper. Les exhortations rageuses de l'assistance à frapper les personnages malveillants sont particulièrement importantes : «Tape-le! achève-le!" L'initié répond habituellement à ces injonctions par le mime des coups à l'aide de son chasse-mouches - le processus de renforcement réciproque entre l'initié et les initiateurs soulignant bien la valeur décisive de la riposte contre le sorcier. Les interventions de l'assistance servent en définitive de support oblique à la relation sorcellaire virtuelle en lui conférant une consistance collective ${ }^{38}$.

Les initiateurs effectuent également un certain nombre de manipulations pour éviter que l'initié ne reste enfermé dans la passivité. Il arrive en effet qu'un banzi ne parvienne pas à sortir de la position de proie impuissante : dans ses visions, il est poursuivi, se sent menacé, tombe dans des trous, est entravé par des liens, se heurte à des 
murs ou se perd dans l'obscurité. Les initiateurs passent alors une torche devant ses yeux et tout autour de lui, afin d'éclairer son chemin et d'en chasser les mauvais esprits. Ou bien ils déchirent devant lui un bout de pagne noir, le geste figurant la levée des obstacles. Si l'impuissance persiste, les initiateurs peuvent aller jusqu'à obliger un tiers - ancien initié ou parent du banzi - à manger l'eboga séance tenante afin qu'il rejoigne dans ses visions le novice en péril et l'aide à sortir de l'impasse. Cela n'empêche pas certaines initiations de rater lamentablement. Ainsi cet homme qui au cours de son initiation avait peu et mal vu. Au matin, il est prostré, l'air complètement abattu, gémissant sur son sort. Le tableau est d'autant plus pitoyable qu'à ses côtés une femme dont l'initiation s'est fort bien passée jubile et exulte bruyamment.

C'est dans ce cadre qu'il faut comprendre la désapprobation unanime des initiateurs à l'égard des pleurs. Il arrive en effet qu'un initié éclate subitement en sanglots, décontenancé par les effets de l'eboga ou accablé par une vision particulièrement malheureuse. Les initiateurs le réprimandent alors aussitôt : "Ici, on ne pleure plus! " Il y ainsi une très nette inversion d'attitude par rapport à la consultation divinatoire dont la réussite était justement marquée par les pleurs du patient. Cette inversion s'explique par la transformation relationnelle opérée dans l'initiation : l'initié doit quitter la position passive qui était encore la sienne lors de la séance divinatoire pour venir occuper une place plus active (voir, raconter ses visions, réagir contre le sorcier). Les pleurs, apitoiement passif sur son mauvais sort, ne sont donc plus tolérables. En définitive, la riposte agressive de l'initié contre le sorcier, dont le mime des coups est la manifestation publique, peut être interprétée comme une inversion des pleurs. Dans le registre des affects, la décharge agressive de l'initiation est un écho inversé des pleurs de la séance divinatoire. L'angoisse impuissante liée à la situation d'infortune est retournée en agressivité dirigée contre le sorcier. Il arrive d'ailleurs que les initiateurs résument une initiation réussie par une expression imagée qui souligne bien l'importance de l'agressivité : «il a bien tapé le ser- 
${ }^{39}$ Le caractère passif ou actif d'un affect est dérivé de la position relationnelle correspon. dante. Un même affect peut donc avoir une valeur différente selon les contextes relation. nels. Les pleurs ne sont pas par nature un affect passif (ainsi les pleurs obligatoires pour certains parents dans les rites funéraires). De même, la décharge agressive n'est pas par nature un affect actif (ainsi l'aveu de faiblesse que constitue une colère inappropriée en public). pent »- le serpent servant ici de métaphore de l'affliction et du sorcier.

L'initiation semble bien déboucher sur une catharsis émotionnelle : "taper le serpent " permet une décharge de tension aussi brutale que libératrice. Ce n'est toutefois pas dans la psychologie des acteurs mais plutôt dans les relations entre ces acteurs qu'il faut chercher la raison des affects. La conversion des pleurs en décharge agressive est la contrepartie directe (à la fois effet et indice) de la dynamique relationnelle du processus initiatique. Mais il ne s'agit pas simplement de saper la relation sorcellaire en se défoulant sur le responsable présumé de l'infortune dans des visions hallucinées. La conversion des affects implique une transformation complexe et cohérente qui concerne autant la structure des interactions concrètes entre acteurs (les relations divinatoire et initiatique) que la mise en scène visionnaire d'une relation virtuelle (la relation sorcellaire). Les transformations dans ces deux sphères - l'une réelle, l'autre imaginaire - obéissent cependant à une même logique générale : il s'agit de convertir un affect passif en un affect actif en faisant passer l'infortuné d'une position de "patient " à une position d' "agent ${ }^{39}$ ". Le patient se trouvait dans une position de proie passive tant dans la relation sorcellaire (proie d'un prédateur cannibale) que dans la relation divinatoire (proie d'un piégeur rusé). L'initiation opère alors une inversion des pôles asymétriques de la relation divinatoire (l'initié en position de devin de soi et des autres) et de la relation sorcellaire (l'initié en position de prédateur omniscient et omnipotent). Et cette double inversion relationnelle se traduit par la jubilation (affect actif dans la relation divinatoire) et surtout la décharge agressive (affect actif dans la relation sorcellaire).

\section{Relations et affects dans les rites d'affliction}

En guise de conclusion, tâchons de mettre en perspective ces résultats ethnographiques dans un cadre plus large. La divination du nganga permet d'introduire le point de vue d'un tiers sur une relation sorcellaire duelle; l'initiation au 
bwete misoko actualise ensuite cette relation virtuelle sur une scène imaginaire - ce que les mimes de l'initié contre son persécuteur donnent directement à voir. Cette mise en scène illustre bien que le rituel est un «drame ${ }^{40}$ ". De cette métaphore théâtrale, il ne faudrait toutefois pas inférer qu'une performance rituelle suit un script écrit à l'avance. En effet, la mise en scène des relations sociales n'est pas une simple représentation conventionnelle de ces relations : il s'y joue véritablement quelque chose. Par rapport aux interactions ordinaires, les interactions rituelles se caractérisent par un degré élevé de réflexivitéét. La communication rituelle fonctionne en effet sur un mode " analogique " plutôt que "digital ${ }^{42}$ ": elle porte davantage sur la relation de communication elle-même que sur des états de chose. Il s'agit toujours de faire saillir des enjeux relationnels au premier plan de l'expérience des acteurs. Or, cette reprise réflexive des relations est la condition même de la performativité du rituel : la mise en scène permet une remise en jeu. Sur ce point, les rites d'affliction ressemblent d'ailleurs singulièrement à la «thérapie systémique " de l'École de Palo Alto ${ }^{43}$; il s'agit d'instaurer un nouveau cadre relationnel (incluant le thérapeute) afin de permettre au patient d'y « jouer " son symptôme de manière réflexive. Ce changement de cadre lui permet alors de redéfinir à partir d'un nouveau point de vue les modèles relationnels (les « métarègles ") sur lesquels il fondait son comportement pathologique (généralement un dilemme relationnel, comme dans les pathologies du couple). De manière significative, le bwete misoko instaure lui aussi un nouveau cadre réflexif (matérialisé par le miroir) permettant de rejouer la relation sorcellaire selon un autre point de vue afin de redéfinir la position qu'occupe l'initié.

Or, c'est justement quand l'enjeu relationnel qui soustend une interaction passe ainsi au premier plan qu'elle a tendance à se charger d'un contenu affectif fort ${ }^{44}$. Voilà pourquoi les émotions occupent habituellement une telle place dans les rituels : un rituel est un enchaînement réglé d'interactions concrètes qui induisent des affects sensibles en actualisant de manière réflexive les enjeux de certaines

\author{
40 Turner, 1982. \\ ${ }^{41}$ Kapferer, 1983; \\ Kordt Højbjerg, 2002; \\ Bonhomme, 2005b. \\ 42 Bateson, 1980. \\ ${ }^{43}$ Watzlawick, 1972. \\ 44 Berthomé, 2006 : \\ $17 \cdot 18$
}


${ }^{45}$ Si le lien entre réflexivité et affect semble être une propriété des situations rituelles, certaines situations non-ritualisées peuvent toutefois être également réflexives et affectivement chargées (rupture ou récon.

ciliation amoureuse par exemple). En outre, la réflexivité n'est pas une condition nécessaire à l'irruption de tous les affects, mais on peut néanmoins penser que la réflexivité favorise l'émergence des affects les plus socialisés, comme c'est habituelle. ment le cas dans le rituel.

${ }^{46}$ Lévi-Strauss, 1958a, 1958b. relations virtuelles particulièrement pertinentes ${ }^{45}$. Cette corrélation entre reprise réflexive des relations et irruption des affects est tout à fait manifeste dans le processus initiatique du bwete misoko. Tout commence par une situation d'affliction qui fait émerger une relation virtuelle et incertaine entre la victime et un sorcier présumé. L'accablement de la victime reflète sa position passive dans cette relation duelle. La consultation divinatoire constitue alors une actualisation indirecte de cette relation virtuelle dans une interaction faisant intervenir un tiers (devin), opération qui confère à la relation sorcellaire un plus grand degré de certitude. Les pleurs du patient trahissent encore sa passivité, mais celleci apparaît désormais sur un mode réflexif (retour sur soi). L'initiation constitue ensuite une actualisation directe de la relation sorcellaire dans une interaction imaginaire (vision) étayée par une interaction réelle entre initié et initiateurs. Jubilation et décharge agressive traduisent alors, sur un mode toujours réflexif, la position désormais active de l'initié. Le schéma d'ensemble (du point de vue de l'infortuné) est donc le suivant : sorcellerie (passivité) $\Rightarrow$ divination (passivité + réflexivité) $\Rightarrow$ initiation (réflexivité + activité).

Cette conversion réflexive d'une position passive en une position active est sans doute une propriété récurrente des rites d'affliction, et plus encore de ceux à caractère initiatique : ces derniers placent en effet le néophyte infortuné au centre des interactions, alors que les soins noninitiatiques en font davantage un "patient ", parfois même absent pendant la cure (guérison "à distance " dans certaines cures chamaniques ou pratiques maraboutiques). Laffliction se caractérisant par une forme d'impuissance passive plutôt que par des symptômes très spécifiques, le retournement de la passivité en activité constitue ainsi une dimension essentielle de la guérison. On a maintes fois souligné que cette "guérison" ne devait pas s'entendre en un sens strictement physiologique : les rites d'affliction possèderaient une efficacité que l'on a pris l'habitude d'appeler "symbolique " ${ }^{46}$, sans toutefois être très clair sur la signification exacte d'un tel épithète. J'espère cependant avoir convaincu le lecteur que l'efficacité propre aux rites 
d'affliction est moins une question de symboles que de relations. Il s'agit par un jeu de transformations relationnelles - qui implique évidemment aussi des manipulations symboliques - de permettre aux infortunés de redevenir actifs. L'irruption des affects constitue alors l'épreuve tangible (pour l'infortuné) et la manifestation publique (pour l'assistance) de la réalité de cette transformation. Les affects passifs qui trahissent la dépossession de la puissance d'agir sont progressivement convertis en affects actifs qui constituent des dispositions à agir, une amorce de réappropriation de la puissance d'agir.

Certes, ce schéma très général se diffracte dans des logiques culturelles singulières qui mobilisent des modèles d'interprétation de l'infortune parfois très différents ${ }^{47}$. La dimension sorcellaire de l'affliction implique ainsi des traits bien spécifiques : la passivité est vécue comme une dépossession par un tiers; la reprise d'initiative passe par une riposte contre ce tiers au prix d'une identification ambivalente avec lui. L'ensemble du parcours initiatique du bwete misoko repose sur ce schéma relationnel singulier. Les étapes initiatiques suivant les rites de passage visent en effet la pérennisation et le renforcement de la position active de l'initié, tant du point de vue de la relation divinatoire que de la relation sorcellaire. D'une part, l'ingestion d'esprits auxiliaires et l'acquisition de fétiches permettent d'activer la parole de l'initié en lui conférant un pouvoir divinatoire non plus temporaire mais permanent. Il s'agit par là de faire passer l'initié du côté du devin, pour qu'il puisse à son tour faire pleurer autrui lors de la consultation. D'autre part, il s'agit de faire définitivement sortir l'initié de la position de victime en le rapprochant, de manière ambivalente, de la position du sorcier. Le processus initiatique est en effet une entreprise d'acquisition et de renforcement du "vampire " (nzanga), terme qui désigne l'organe de sorcellerie, puissance logée dans le ventre du sorcier qui lui permet d'agresser ses victimes la nuit venue. Or, une pratique assidue du bwete permet de "réveiller", "mettre debout", "soulever " ou " faire monter » le vampire (il est révélateur que ce soient des verbes d'action). Un initié accompli doit ainsi posséder

${ }^{47}$ Augé \& Herzlich, 1984. 
${ }^{48}$ Favret-Saada \& Contreras, 1985.

${ }^{49}$ Kapferer, 1979, 1983. un nzanga fort afin de conférer détermination et assurance à ses actes et à ses paroles. La transformation initiatique repose donc sur l'acquisition d'une puissance d'agir directement associée à la sorcellerie : « Il n'y a [en effet] que les vampireux qui réussissent. " Redevenir actif implique en définitive que l'initié s'approprie l'agressivité au principe de la relation sorcellaire.

Ce travail ambivalent sur l'agressivité est sans doute un trait récurrent des rites de contre-sorcellerie ${ }^{48}$. Il ne représente cependant qu'une actualisation parmi d'autres du schéma relationnel d'ensemble des rites d'affliction. Les rites d'exorcisme au Sri Lanka empruntent par exemple une autre voie ${ }^{49}$. L'affliction est attribuée à un démon que le rituel met en scène au cours d'une inquiétante mascarade qui vise à susciter la peur du patient. À cette phase dramatique de l'exorcisme succède une phase comique : le démon devient un être ridicule que les hommes raillent et humilient (jusqu'à parfois le faire pleurer!). L'enjeu est alors d'amener le patient à se joindre à l'hilarité de l'assistance. La victime doit en effet parvenir à rire de sa propre peur, qui lui apparaît désormais aussi grotesque que le démon luimême. L'exorcisme permet ainsi de convertir la peur (affect passif) en un rire éminemment réflexif (affect actif). Ces rires opèrent donc une forme de catharsis assez différente de la décharge agressive du bwete misoko. Ces différents affects traduisent ainsi deux modes distincts de l'inversion de la relation au principe de l'affliction : dans un cas, la réappropriation du pouvoir (empowerment) de la victime passe par une identification ambivalente à l'agresseur; dans l'autre cas, la réappropriation repose sur une dévaluation du pouvoir (disempowerment) de l'agresseur tourné en ridicule. Si le schéma d'ensemble est identique, les modalités de son accomplissement diffèrent donc sensiblement. Mais seul un inventaire comparatiste plus systématique permettra de dégager le répertoire des variations possibles autour de ce schéma général de la conversion relationnelle et affective d'une impuissance passive en une puissance active. Quels sont les modes affectifs de la passivité? Et comment peut-on redevenir actif? 


\section{Références bibliographiques}

Augé, M. \& C. Herzlich (dir.)

1984 Le Sens du mal. Anthropologie, histoire, sociologie de la maladie, Paris, Éditions des Archives Contemporaines.

Austen, R.

1993 "The moral economy of witchcraft : an essay in comparative history ", in J. \& J. L. Comaroff (dir.), Modernity and its malcontents. Ritual and power in postcolonial Africa, Chicago, The University of Chicago Press.

Bateson, G.

1977 "La cybernétique du "soi”. Une théorie de l'alcoolisme ", in Vers une écologie de l'esprit, Paris, Seuil, T. 1,.

1980 "Les problèmes de communication chez les cétacés et autres mammifères ", in Vers une écologie de l'esprit, Paris, Seuil, T. 2.

1991 "Un regard des sciences sociales sur les émotions", in Une unité sacrée, Paris, Seuil.

Berthomé, F.

2006 Etre affecté. Relations, interactions et émotions dans quelques rituels mélanésiens, mémoire de DEA en anthropologie, Paris, EHESS.

Bonhomme, J.

2005a "Voir par-derrière. Sorcellerie, initiation et perception au Gabon ", Social Anthropology 13 (3), pp. 259-273.

2005b Le Miroir et le Crâne. Parcours initiatique du bwete misoko (Gabon), Paris, CNRS Éditions.

Csordas, T. J. (dir.)

1994 Embodiment and experience. The existential ground of culture and self, Cambridge, Cambridge University Press.

Deshayes, P.

2002 "Frayeurs et visions chamaniques: le malentendu thérapeutique ", Psychologie française 47 (4), pp. 5-14.

Favret-Saada, J.

1977 Les Mots, la mort, les sorts, Paris, Gallimard.

1990 "Etre affecté », Gradhiva 8, pp. 3-9.

Favret-Saada, J. \& J. Contreras

1985 "L'embrayeur de violence. Quelques mécanismes thérapeutiques du désorcèlement ", in M. Mannoni (dir.), Le Moi et l'Autre, Paris, Denoël,

Gell, A. pp. 95-125.

1998 Art and agency. An anthropological theory, Oxford University Press. 
Geschiere, P.

1995 Sorcellerie et politique en Afrique. La viande des autres, Paris, Karthala.

Gluckman, M.

1963 "Rituals of rebellion in south-east Africa " in Order and rebellion in tribal Africa, Londres, Cohen \& West.

Houseman, M.

1986 "Le mal pour le mâle: un bien initiatique ", in J. Hainard \& R. Kaehr (dir.), Le Mal et la douleur, Neuchâtel, Musée d'ethnographie, pp. 89-99.

2002 "Dissimulation and simulation as forms of religious reflexivity ", Social Anthropology 10(1-2), pp. 77-89.

2003 "Vers un modèle anthropologique de la pratique psychothérapeutique ", Thérapie familiale 24(3), pp. 289-312.

2006 "Relationality ", in J. Kreinath, J. Snoek \& M. Stausberg (dir.), Theorizing rituals. Issues, topics, approaches, concepts, Leiden, Brill, pp. 413-428.

Houseman, M. \& C. Severi

1994 Naven ou le donner à voir. Essai d'interprétation de l'action rituelle, Paris, CNRS Éditions.

Kapferer, B.

1979 "Emotion and feeling in Sinhalese healing rites", Social Analysis 1, pp. $153-176$

1983 A celebration of demons. Exorcism and the aesthetics of healing in Sri Lanka, Bloomington, Indiana University Press.

1997 The Feast of the sorcerer. Practices of consciousness and power, Chicago, The University of Chicago Press.

Kordt Højbjerg, C. (dir.)

2002 "Religious reflexivity", Social Anthropology 10 (1-2).

Lévi-Strauss, C.

1958a "Le sorcier et sa magie ", in Anthropologie structurale, Paris, Plon.

1958 b "L'efficacité symbolique ", in Anthropologie structurale, Paris, Plon.

1971 L'Homme nu. Mythologiques 4, Paris, Plon.

Lewis, I. M.

1971 Ecstatic religion. An anthropological study of spirit possession and shamanism,

Scheff, T. J. Harmondsworth, Penguin Books.

1977 "The distancing of emotion in ritual », Current Anthropology 18(3), pp. 483. 505.

1979 Catharsis in healing, ritual and drama, Berkeley, University of California Press.

Schieffelin, E. L.

1985 "Performance and the cultural construction of reality", American Ethnologist 12(4), pp. 707-724. 
Spinoza

1988 Éthique, Paris, Seuil [trad. B. Pautrat].

Taylor, A.-C.

1993 "Des fantômes stupéfiants. Langage et croyance dans la pensée achuar ", L'Homme 126-128, pp. 429-447.

Turner, V. W.

1968 The Drums of affliction. A study of religious process among the Ndembu of Zambia, Oxford, Clarendon Press.

1969 The Ritual process. Structure and anti-structure, Chicago, Aldine.

1982 "Dramatic ritual / ritual drama. Performative and reflexive anthropology ", in From ritual to theatre, New York, Performing Arts Journal Publications.

Watzlawick, P. \& J. Helmick Beavin, D. D. Jackson

1972 Une logique de la communication, Paris, Seuil, 1972.

Whitehouse, H.

2000 Arguments and icons. Divergent modes of religiosity, Oxford, Oxford University Press.

Zempléni, A.

1985 "La maladie et ses causes ", L'Ethnographie 81 (96-97), pp. 13-44. 